\title{
Tendência temporal da mortalidade por Câncer de Colo do Útero em Sergipe
}

\author{
Temporal trend of Cervical Cancer mortality in Sergipe \\ Tendencia temporal de mortalidad por Cáncer de Cuello Uterino en Sergipe
}

Recebido: 05/01/2021 | Revisado: 08/01/2021 | Aceito: 12/01/2021 | Publicado: 13/01/2021

\author{
Manuela de Carvalho Vieira Martins \\ ORCID: https://orcid.org/0000-0003-1222-5955 \\ Universidade Tiradentes, Brasil \\ E-mail: manuela.cvm@ hotmail.com \\ Késsia Raianne Santos Carregosa \\ ORCID: https://orcid.org/0000-0001-8259-2668 \\ Universidade Tiradentes, Brasil \\ E-mail: kessia-santos@hotmail.com \\ Marcelly Conceição Menezes Anchieta \\ ORCID: https://orcid.org/0000-0001-7753-4172 \\ Universidade Tiradentes, Brasil \\ E-mail: marcellyanchieta04@gmail.com \\ Jefferson Felipe Calazans Batista \\ ORCID: https://orcid.org/0000-0002-3681-7990 \\ Universidade Tiradentes, Brasil \\ E-mail: jefferson.calazans.enf@gmail.com \\ Thandara Rejane Santos Ferreira Andrade \\ ORCID: https://orcid.org/0000-0002-3385-7727 \\ Universidade Tiradentes, Brasil \\ E-mail: thandara456@outlook.com \\ Ianka Heloisa Alencar Santos \\ ORCID: https://orcid.org/0000-0002-2057-0727 \\ Universidade Tiradentes, Brasil \\ E-mail: iankaheloisa@ outlook.com \\ Yasmim Anayr Costa Ferrari \\ ORCID: https://orcid.org/0000-0003-1766-341X \\ Universidade Tiradentes, Brasil \\ E-mail: yasmimanayr@hotmail.com \\ Carla Viviane Freitas de Jesus \\ ORCID: https://orcid.org/0000-0002-7775-6610 \\ Universidade Tiradentes, Brasil \\ E-mail: carlavfj@gmail.com \\ Rebecca Maria Oliveira de Góis \\ ORCID: https://orcid.org/0000-0002-3935-5904 \\ Universidade Tiradentes, Brasil \\ E-mail: rebecca.gois@ hotmail.com \\ Fernanda Costa Martins Galotti \\ ORCID: https://orcid.org/0000-0002-9063-1273 \\ Universidade Tiradentes, Brasil \\ E-mail: fercosmart@gmail.com \\ Sonia Oliveira Lima \\ ORCID: https://orcid.org/0000-0002-3257-2412 \\ Universidade Tiradentes, Brasil \\ E-mail: sonialima.cirurgia@gmail.com
}

\begin{abstract}
Resumo
Objetivo: Analisar a tendência temporal da mortalidade por câncer de colo de útero em Sergipe, no período de 2000 a 2018, bem como identificar sua relação com taxa de cobertura de exames citopatológicos. Metodologia: Trata-se de um estudo ecológico de séries temporais, cujos dados referentes à população feminina residente, número de óbitos por neoplasia maligna de colo de útero e número de exames citopatológicos realizados, estratificados por faixa etária e outras variáveis socioeconômicas na Unidade da Federação e período descritos, foram extraídos do DATASUS e de seus sistemas associados. Resultados: Foram verificados, entre 2000 e 2018, 1.239 óbitos por câncer de colo de útero em Sergipe, dos quais 23,18\% eram mulheres de 50 a 59 anos; $51,49 \%$ de cor parda; $24,53 \%$ com nenhuma escolaridade; $40,36 \%$ solteiras e $67,71 \%$ ocorreram em ambiente hospitalar. Na cobertura de exames citopatológicos, foram encontrados dados de 2006 a 2018, identificou-se 1.067 .695 exames realizados no mesmo local, a maioria possuía raça/cor, escolaridade, estado civil e ocorrência ignorados ou não registrados e 27,80\% eram mulheres de 20 a 29 anos. Conclusão: Percebe-se a tendência de crescimento da mortalidade e a tendência de diminuição da taxa de cobertura, fato que, mesmo não significativo estatisticamente, pode representar variáveis inversamente proporcionais.
\end{abstract}


Palavras-chave: Neoplasias do colo do útero; Estudos de séries temporais; Mortalidade; Saúde da mulher; Sistemas de informação em saúde.

\begin{abstract}
Objective: To analyze the temporal trend of cervical cancer mortality in Sergipe, from 2000 to 2018, as well as to identify its relationship with the coverage rate of cytopathological tests. Methodology: This is an ecological study of time series, whose data referring to the resident female population, number of deaths from malignant neoplasia of the cervix and number of cytopathological tests performed, stratified by age group and other socioeconomic variables in the Federation Unit and period described, were extracted from DATASUS and its associated systems. Results: Between 2000 and 2018, 1,239 deaths from cervical cancer were verified in Sergipe, of which 23.18\% were women aged 50 to 59 years; $51.49 \%$ brown color; $24.53 \%$ with no schooling; $40.36 \%$ single and $67.71 \%$ occurred in the hospital environment. In the coverage of cytopathological tests, data were found from 2006 to 2018, 1,067,695 tests performed at the same site were identified, most had race/color, schooling, marital status and occurrence ignored or unregistered, and $27.80 \%$ were women aged 20 to 29 years. Conclusion: The trend of mortality growth and the trend of decrease in the coverage rate are perceived, a fact that, even if not statistically significant, may represent inversely proportional variables.
\end{abstract}

Keywords: Uterine cervical neoplasms; Time series studies; Mortality; Women's health; Health information systems.

\title{
Resumen
}

Objetivo: Analizar la tendencia temporal de mortalidad por cáncer de cuello uterino en Sergipe, de 2000 a 2018 , así como identificar su relación con la tasa de cobertura de las pruebas citopáticas. Metodología: Se trata de un estudio ecológico de series temporales, cuyos datos referentes a la población femenina residente, número de muertes por neoplasia maligna del cuello uterino y número de pruebas citopatológicas realizadas, estratificadas por grupo de edad y otras variables socioeconómicas en la Unidad de Federación y período descrito, fueron extraídos de DATASUS y sus sistemas asociados. Resultados: Entre 2000 y 2018, se verificaron 1.239 muertes por cáncer de cuello uterino en Sergipe, de las cuales el 23,18\% eran mujeres de 50 a 59 años; $51.49 \%$ color marrón; 24,53\% sin escolarización; El $40,36 \%$ solo y el $67,71 \%$ ocurrieron en el entorno hospitalario. En la cobertura de las pruebas citopatológicas, se encontraron datos de 2006 a 2018, se identificaron 1.067.695 pruebas realizadas en el mismo sitio, la mayoría tenía raza/color, escolaridad, estado civil e ocurrencia ignoradas o no registradas, y el 27,80\% eran mujeres de 20 a 29 años. Conclusión: Se percibe la tendencia de crecimiento de la mortalidad y la tendencia de disminución de la tasa de cobertura, un hecho que, aunque no sea estadísticamente significativo, puede representar variables inversamente proporcionales.

Palabras clave: Neoplasias del cuello uterino; Estudios de series temporales; Mortalidad; Salud de la mujer; Sistemas de información en salud.

\section{Introdução}

O câncer do colo do útero é o terceiro tumor maligno mais frequente na população feminina, atrás do câncer de mama e do colorretal, sendo a incidência dessa patologia nas mulheres brasileiras uma das mais altas do mundo. Além disso, é a quarta causa de morte de mulheres por câncer no Brasil (Inca, 2020a). Este tipo de câncer apresenta um grande impacto nos índices de morbidade e mortalidade, podendo ser minimizado por meio da detecção precoce (Carvalho, 2019).

Além dos impactos citados anteriormente, o câncer de colo de útero gera alterações bastante significativas na qualidade de vida das mulheres. A título de exemplo, temos as lesões funcionais secundárias aos tratamentos, como as decorrentes da radioterapia e da remoção de partes da anatomia genital feminina em cirurgias pélvicas; os efeitos colaterais da quimioterapia, que, em parte, são comuns aos da radioterapia, como náusea, vômito, diarreia, constipação, mudanças de peso e alterações hormonais; os fatores psicológicos, que incluem crenças erradas sobre a origem do câncer, alterações na autoimagem, baixa autoestima, disfunção sexual, tensões matrimoniais, medos e preocupações (Correia et al., 2018; Silva et al., 2018).

O surgimento do câncer se dá através do crescimento desordenado do epitélio do colo do útero, que por ter desenvolvimento lento, pode ocorrer sem apresentar sintomas evidentes em sua fase inicial. Contudo, quando presentes, nesse estágio inicial, apresentam-se por meio de sintomas ginecológicos relacionados às doenças infecciosas, como corrimento, prurido e dor. Por outro lado, manifestações mais graves como sangramento vaginal e corrimento de odor fétido podem surgir no estágio mais avançado da neoplasia, o que dificulta a cura devido ao diagnóstico tardio (Carvalho et al., 2018). 
Grande parte das mulheres diagnosticadas com essa neoplasia, apesar de cientes da importância de seguir com o tratamento após a detecção de lesões precursoras, não aderem às orientações recebidas, pois, a percepção sobre a prevenção está associada às relações com os serviços de saúde, familiares e a fatores sociodemográficos (Leite et al., 2018).

No mundo, em 2012, o câncer do colo do útero foi responsável por mais de 265 mil óbitos em mulheres, sendo que 87\% desses óbitos ocorreram em países em desenvolvimento. Estima-se que no ano de 2020 surjam aproximadamente 16.590 novos casos e 6.526 mortes no Brasil (Inca, 2020b). A sobrevida vem obtendo melhora ao longo dos anos, variando de menos de 50\% para mais de 70\% em todo o mundo (Febrasgo, 2017).

O maior fator de risco para desenvolvimento de câncer do colo do útero é o Papiloma Vírus Humano (HPV), especialmente o HPV-16 e o HPV-18. A infecção por esse vírus é muito comum, visto que cerca de $80 \%$ das mulheres sexualmente ativas irão adquiri-la ao longo de suas vidas, e, na maioria das vezes, retrocede espontaneamente, entre seis meses a dois anos após a exposição. Apesar de ser uma condição de saúde notória, a infecção pelo HPV, isoladamente, é insuficiente para o desenvolvimento da neoplasia, o que sugere que outros fatores também estejam envolvidos no surgimento do câncer, como o uso prolongado de pílulas anticoncepcionais, tabagismo, início precoce da atividade sexual e múltiplos parceiros (Inca, 2020a).

O câncer do colo uterino é considerado, de uma forma geral, uma neoplasia de bom prognóstico, desde que seja diagnosticado de forma precoce e tratado de maneira apropriada (Inca, 2016). Uma eficiente medida para identificação prévia da doença e, consequentemente, redução da mortalidade é o rastreamento extensivo e incessante através do exame citopatológico. Essa simples ação pode favorecer a redução de óbitos por câncer de colo de útero em mais de $70 \%$ em relação às populações não assistidas. O propósito desse rastreamento é detectar alterações celulares em estádios iniciais para que as medidas de intervenção ideais sejam sancionadas (Farias \& Barbieri, 2016).

Dessa maneira, compreendendo o câncer de colo do útero como um expressivo problema de saúde pública, atenta-se para a necessidade de se avaliar os índices de mortalidade dessa doença para que medidas consonantes sejam adotadas e permitam a minimização desses índices. Para tanto, este trabalho objetivou analisar a tendência temporal da mortalidade por câncer de colo de útero no Estado de Sergipe, no período de 2000 a 2018, bem como identificar sua relação com a taxa de cobertura de exames citopatológicos.

\section{Metodologia}

Trata-se de um estudo ecológico de séries temporais sobre a mortalidade de câncer de colo de útero no Estado de Sergipe, no período de 2000 a 2018. Essa unidade da federação localizada no Nordeste brasileiro, é composta por 75 municípios, divididos em 7 regiões de saúde e em 13 microrregiões. O Estado de Sergipe ocupa uma área de $21910 \mathrm{~km}^{2}$ e possui, em 2020, uma população estimada de 2.534 .193 habitantes.

O presente estudo possui caráter quantitativo, pois pode ser analisado por meio de técnicas matemáticas, o que permite a previsão de inúmeras possibilidades acerca da temática abordada (Pereira et al., 2018). Para realizar a análise temporal da taxa de mortalidade por câncer de colo de útero, buscou-se compreender o perfil sociodemográfico das vítimas dessa doença no Estado de Sergipe, assim como, a evolução dos percentuais dessa patologia ao longo dos anos, através da inclusão de variáveis sociodemográficas (faixa etária, raça/cor, estado civil, escolaridade, local de ocorrência) e taxa de mortalidade por faixa etária (<20 anos, 20-30 anos, 40-49 anos, 50-59 anos, 60-69 anos, 70-79 anos e 80 anos ou mais).

A coleta de dados foi realizada através do Portal do Departamento de Informática do Sistema Único de Saúde (DATASUS), disponibilizado pelo Ministério da Saúde do Brasil. O DATASUS agrega informações de censos demográficos e estimativas populacionais realizadas pelo Instituto Brasileiro de Geografia e Estatística (IBGE) a dados do Sistema de Informações sobre Mortalidade (SIM), do Sistema de Informação do Câncer (SISCAN) e do Sistema de Informação do câncer 
do colo do útero (SISCOLO), bem como aqueles provenientes de outros sistemas.

Para a coleta de dados referentes à mortalidade, foram considerados os seguintes critérios de inclusão: 1) Unidade da Federação: Sergipe; 2) Categoria do CID-10: C53 - neoplasia maligna de colo de útero; 3) Sexo: feminino; 4) Período 2000 2018. Esses dados também foram estratificados de acordo com as variáveis sociodemográficas em estudo.

Os dados referentes a cobertura dos exames citopatológicos cérvico-vaginal e microflora foram coletadas no SISCOLO (versão 4.0) para o período de 2006 a 2012, porém devido a indisponibilidade das informações para o período em estudo, os demais dados foram coletados no SISCAN para o período de 2013 a 2018. Em ambos os portais não estavam disponíveis tais informações para o período de 2000 a 2005. Para essa busca, foram utilizados os seguintes critérios de inclusão: 1) Unidade da Federação: Sergipe;2) Cito do colo - por local de residência; 3) Período 2006-2018. Esses dados também foram estratificados de acordo com as variáveis em estudo.

Ademais, dados populacionais foram coletados para a mesma Unidade da Federação, Sexo e Período. Contudo, devido à indisponibilidade de dados provenientes de Censos e Projeções intercensitárias após 2012, as informações dos anos de 2013 a 2018, foram coletadas através da Projeção da População das Unidades da Federação, também disponibilizada no DATASUS.

Quadro 1. Taxa de mortalidade anual do Estado (TMA) para 100 mil habitantes. $T M A=\frac{\text { № de óbitos por neoplasia maligna de colo de útero em Sergipe no período }}{\text { População feminina no mesmo local e período }} \times 100 \mathrm{mil}$ Fonte: Autores.

De acordo com o Quadro 1, foram calculadas para 100 mil habitantes a Taxa de mortalidade anual do Estado.

Quadro 2. Taxa de mortalidade no período (TMP) para 100 mil habitantes.

$$
\begin{aligned}
& \text { TMP } \\
& =\frac{\text { Média de óbitos }(2000-2018) \text { por neoplasia maligna de colo de útero em Sergipe }}{\text { População feminina no meio do período no local }} \times 100 \text { mil }
\end{aligned}
$$

Fonte: Autores.

No tocante ao Quadro 2, abordou-se o cálculo da Taxa de mortalidade do período.

Quadro 3. Taxa de mortalidade segundo faixa etária (TMFE) para 100 mil habitantes.

$$
\begin{aligned}
& \text { TMFE } \\
& =\frac{\mathrm{N} \text { o óbitos por neoplasia maligna em mulheres no local e ano em cada faixa etária }}{\text { População feminina no local e ano em cada faixa etária }} \times 100 \text { mil }
\end{aligned}
$$

Em relação ao Quadro 3, obteve-se a Taxa de mortalidade segundo faixa etária. 
Quadro 4. Taxa de cobertura de exames citopatológicos anual do Estado (TCA) (\%).

$T C A=\frac{\text { № de exames citopatológicos em Sergipe no período }}{\text { População feminina no mesmo local e período }} x 100$

Fonte: Autores.

Além disso, no Quadro 4 foram calculadas a Taxa de cobertura de exames citopatológicos anual do Estado.

Quadro 5. Taxa de cobertura de exames citopatológicos do período (TCP) (\%).

$T C P=\frac{\text { Média de exames citopatológicos em Sergipe no período de 2006 a 2018 }}{\text { População feminina no meio do período no local }} x 100$

Fonte: Autores.

Quanto ao Quadro 5, foram calculadas e analisadas a Taxa de cobertura de exames citopatológicos do período.

Quadro 6. Taxa de cobertura de exames citopatológicos segundo faixa etária (TCFE) (\%).

$T C F E=\frac{N \text { exames citopatoló gios no local e ano em cada faixa etária }}{\text { População feminina no local e ano em cada faixa etária }} 100$

Fonte: Autores.

De acordo, com o Quadro 6, foi calculado a taxa de cobertura de exames citopatológicos segundo faixa etária, todas expressas em porcentagem.

A taxa de mortalidade foi padronizada pelo método direto, utilizando a população feminina brasileira como referência e considerando a seguinte estrutura etária: 0-4, 5-9, 10-14, 15-19, 20-29, 30-39, 40-49, 50-59, 60-69, 70-79, 80 ou mais. A caracterização sociodemográfica da população em estudo foi apresentada por meio de frequências absolutas e relativas, referentes ao número de óbitos e à cobertura de exames citopatológicos em Sergipe.

Para a análise de série temporal, foi utilizado o modelo de regressão por pontos de inflexão (joinpoint regression model). Esse modelo permite identificar se uma linha com múltiplos segmentos é mais apropriada estatisticamente para retratar a evolução temporal de um conjunto de dados quando comparada a uma linha reta ou a menos segmentos. Calcularam- se o percentual de variação anual (APC, do inglês Anual Percent Change) e o percentual de variação médio do período (AAPC, do inglês Average Anual Percent Change), com intervalo de confiança de 95\% (IC 95\%) e a significância de 5\%.As tendências foram classificadas em crescente, decrescente ou estacionária conforme avaliação do APC do segmento, AAPC do período total e sinal do IC95\%. A análise foi realizada utilizando o programa Joinpoint, versão 4.8.0.1 (Surveillance Research, National Cancer Institute, USA).

Por utilizar dados secundários de domínio público, este estudo não foi submetido à apreciação pelo Comitê de Ética em Pesquisa. 


\section{Resultados}

Tabela 1. Perfil sociodemográfico dos óbitos femininos por neoplasia maligna de colo de útero em Sergipe, Brasil para o período de 2000-2018 e dos exames citopatológicos realizados no mesmo local no período de 2006-2018.

\begin{tabular}{|c|c|c|c|c|}
\hline Variáveis & & & & \\
\hline Faixa etária & $\mathrm{N}^{\mathbf{0}}$ de óbitos & \% de óbitos & $N^{0}$ de exames & $\%$ de exames \\
\hline Até 14 anos & 0 & 0,00 & 6072 & 0,57 \\
\hline 15 a 19 anos & 1 & 0,08 & 75874 & 7,11 \\
\hline 20 a 29 anos & 37 & 2,99 & 29678 & 27,80 \\
\hline 30 a 39 anos & 145 & 11,71 & 290735 & 27,23 \\
\hline 40 a 49 anos & 272 & 21,97 & 211251 & 19,79 \\
\hline 50 a 59 anos & 287 & 23,18 & 122283 & 11,45 \\
\hline 60 a 69 anos & 221 & 17,85 & & \\
\hline 70 a 79 anos & 169 & 13,65 & $64693 *$ & 6,06 \\
\hline 80 anos ou mais & 106 & 8,56 & & \\
\hline Ignorado & 1 & 0,08 & 9 & 0,00 \\
\hline
\end{tabular}

\begin{tabular}{|c|c|c|c|c|}
\hline Cor/raça & $\mathrm{N}^{0}$ de óbitos & \% de óbitos & $N^{0}$ de exames & $\%$ de exames \\
\hline Branca & 299 & 24,13 & 1395 & 0,13 \\
\hline Preta & 100 & 8,07 & 2402 & 0,22 \\
\hline Amarela & 4 & 0,32 & 344 & 0,03 \\
\hline Parda & 638 & 51,49 & 70861 & 6,64 \\
\hline Ignorado & 198 & 15,98 & 985983 & 92,35 \\
\hline Escolaridade & $N^{0}$ de óbitos & \% de óbitos & $\mathbf{N}^{0}$ de exames & \% de exames \\
\hline Nenhuma & 304 & 24,53 & 14858 & 1,39 \\
\hline 1 a 3 anos & 277 & 22,35 & \multirow{2}{*}{$85663 *$} & \multirow{2}{*}{8,02} \\
\hline 4 a 7 anos & 200 & 16,14 & & \\
\hline 8 a 11 anos & 110 & 8,87 & 35368 & 3,31 \\
\hline 12 anos e mais & 29 & 2,34 & 26951 & 2,52 \\
\hline Ignorado & 319 & 25,74 & 904855 & 84,75 \\
\hline
\end{tabular}

Legenda: * valores mesclados devido a indisponibilidade de dados estratificados. - dados indisponíveis nos sistemas de busca utilizados.

Fonte: DATASUS (2020).

No período em estudo, foram identificados 1.239 óbitos por neoplasia maligna de colo de útero em mulheres no Estado de Sergipe, dos quais 23,18\% (n=287) foram de mulheres na faixa etária de 50-59 anos; 51,49\% de cor parda; 25,74\% escolaridade ignorada, seguida de $24,53 \%$ de mulheres com nenhuma escolaridade; $40,36 \%$ solteiras e $67,71 \%$ de óbitos em 
ambiente hospitalar (Tabela 1). Não foram identificados óbitos em menores de 15 anos.

No mesmo intervalo, foram verificados 1.067.695 exames citopatológicos realizados no Estado de Sergipe, dos quais 27,80\% foram de mulheres na faixa etária de 20 a 29 anos; 92,35\% de raça/cor ignorada, seguida de 6,64\% de raça/cor parda; $84,75 \%$ de escolaridade ignorada seguido por 8,02\% de escolaridade entre 1 e 7 anos (Tabela 1). Não foram encontrados dados especificados para as variáveis "Estado civil" e "Local de ocorrência".

Quadro 7. Taxa de mortalidade anual (TMA) de neoplasia maligna de útero em Sergipe, Brasil, 2000-2018.

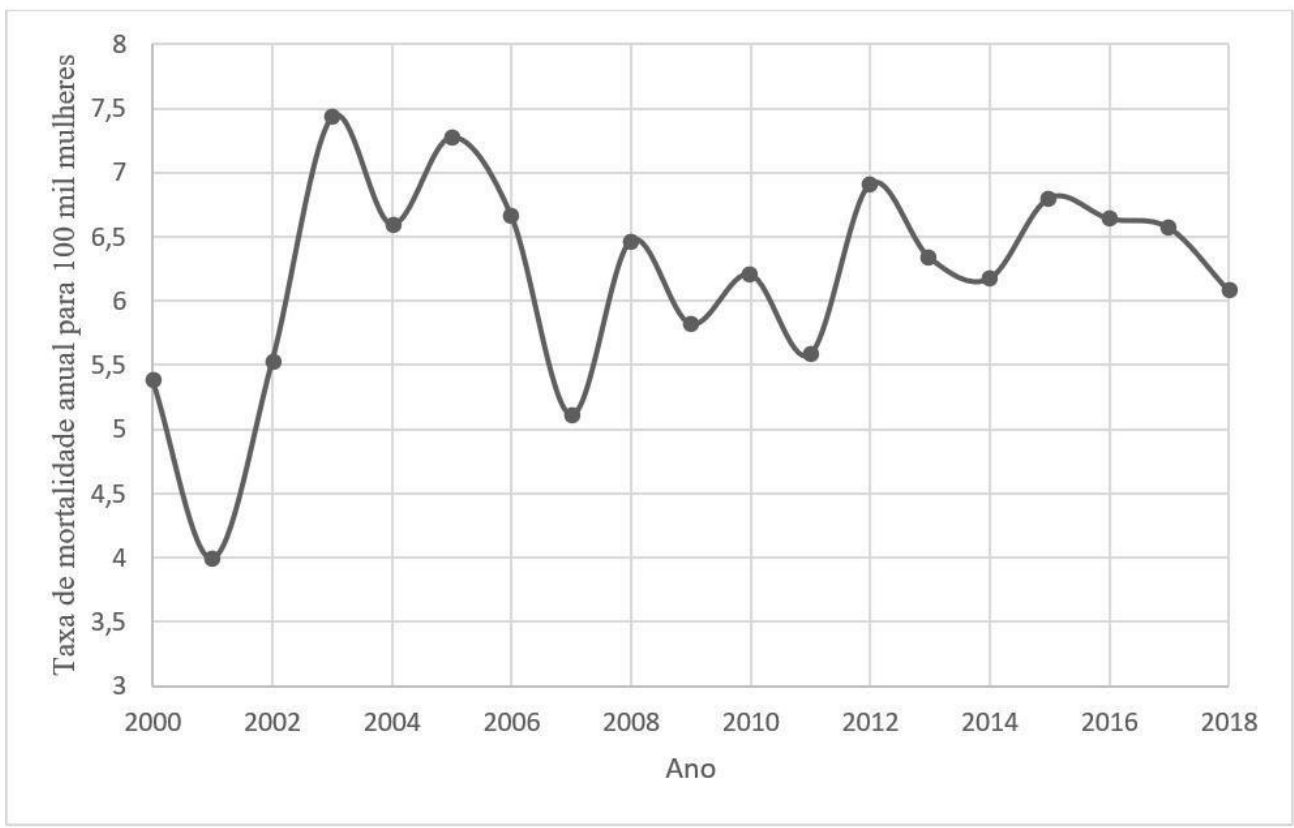

Fonte: DATASUS (2020).

Em relação ao Quadro 7, a taxa de mortalidade anual (TMA) foi calculada para o período de 2000 a 2018 e expressa no Quadro 7. Nela, é possível observar, para cada 100 mil mulheres, a TMA inicial de 5,38 óbitos; TMA final de 6,08 óbitos; TMA máxima de 7,4 óbitos e TMA mínima de 4 óbitos. A Taxa de mortalidade do período (TMP) também foi obtida, indicando 6,33 óbitos para cada 100 mil mulheres. 
Research, Society and Development, v. 10, n. 1, e29510111680, 2021

(CC BY 4.0) | ISSN 2525-3409 | DOI: http://dx.doi.org/10.33448/rsd-v10i1.11680

Quadro 8. Taxa de cobertura anual (TCA) de exames citopatológicos realizados em Sergipe, Brasil, 2006-2018.

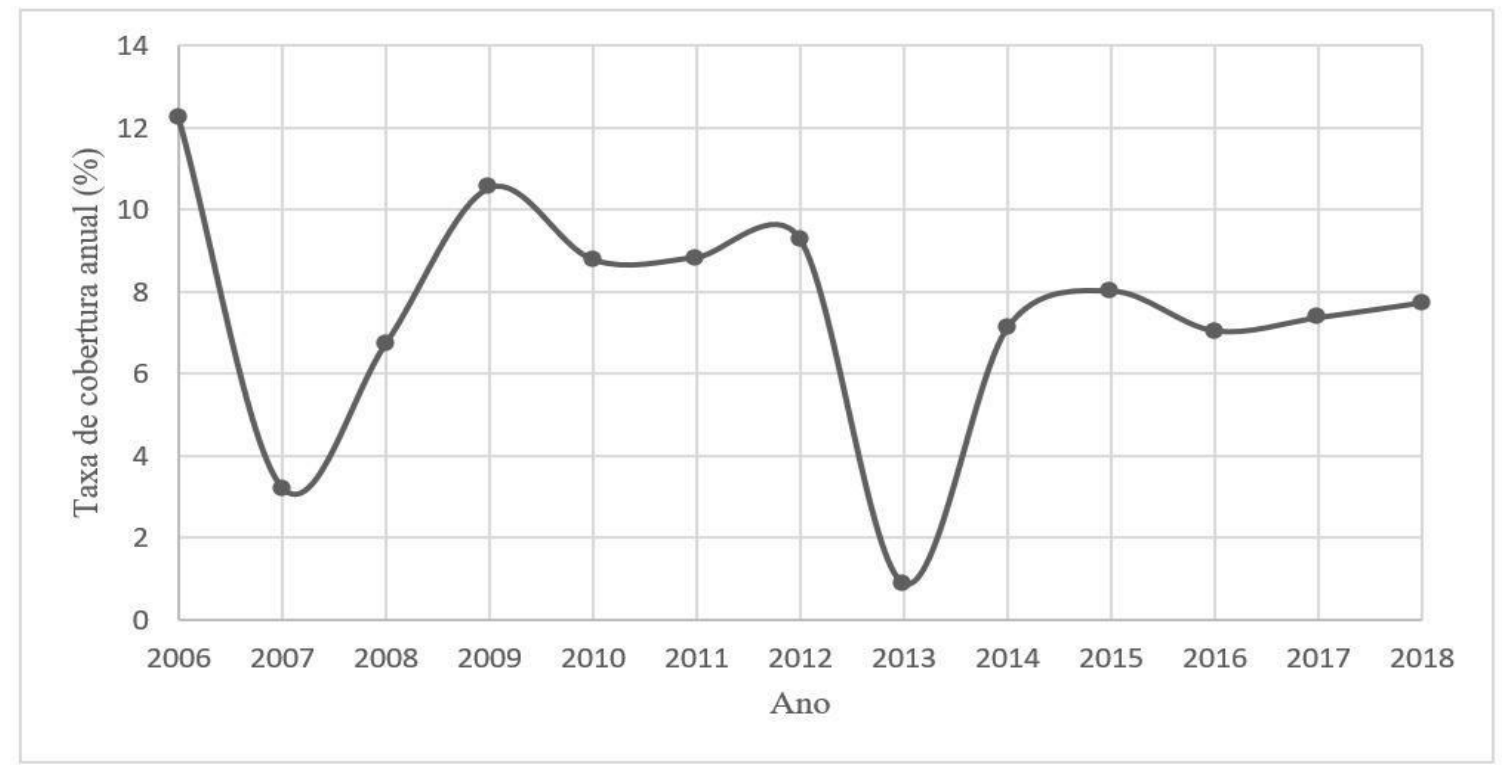

Fonte: DATASUS (2020).

Segundo o Quadro 8, A taxa de cobertura anual de exames citopatológicos (TCA) também foi calculada, porém, o período utilizado foi o de 2006 a 2018 devido a indisponibilidade dos dados dos anos anteriores. Nela, é possível observar a TCA inicial e máxima de 12,24\%, TCA final de 7,34\% e TCA mínima de 0,87\%. A Taxa de cobertura do período (2006 a 2018) também foi obtida, indicando o total de $7,57 \%$. 
Quadro 9. Taxa de mortalidade por neoplasia maligna de colo de útero a cada 100 mil mulheres em Sergipe, Brasil, 20002018.

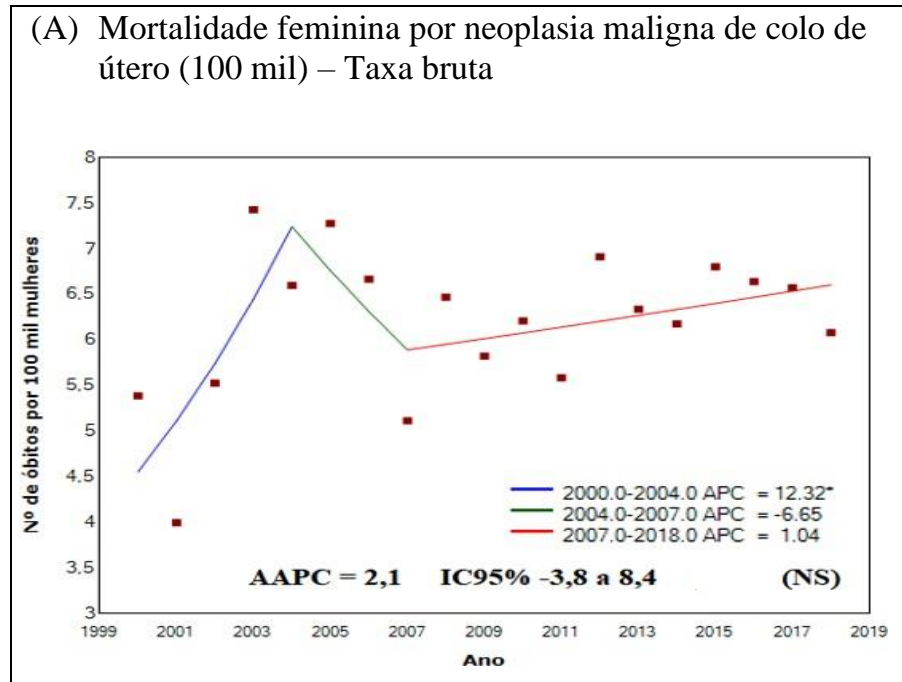

(C) TMFE 20 a 29 anos

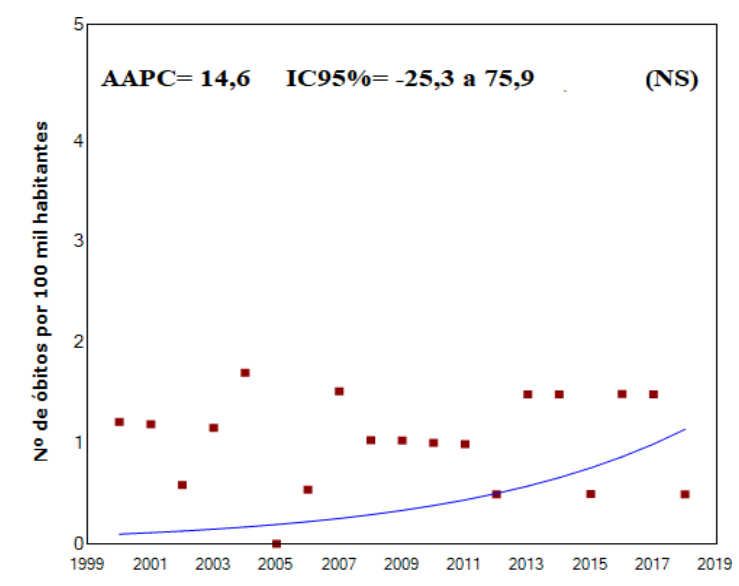

(E) TMFE 40 a 49 anos

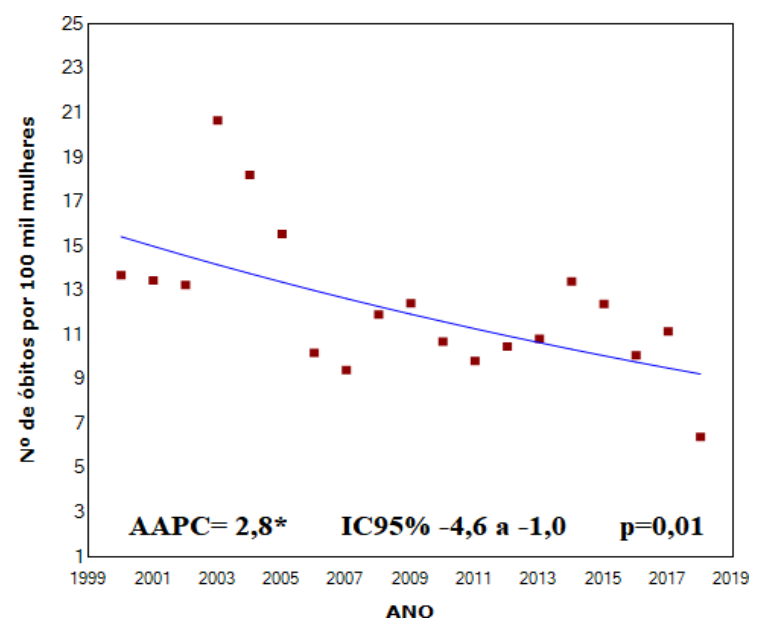

(B) Mortalidade feminina por neoplasia maligna de colo de útero (100 mil) - Taxa padronizada

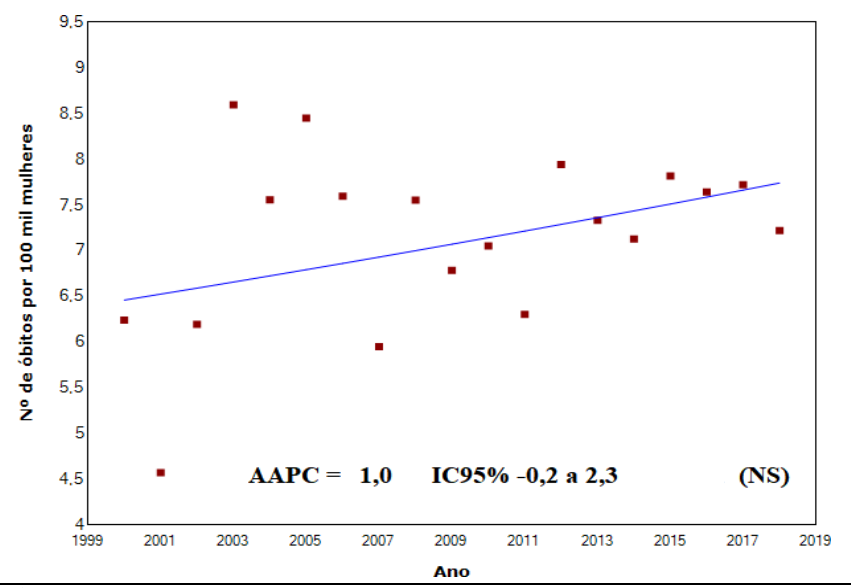

(D) TMFE 30 a 39 anos

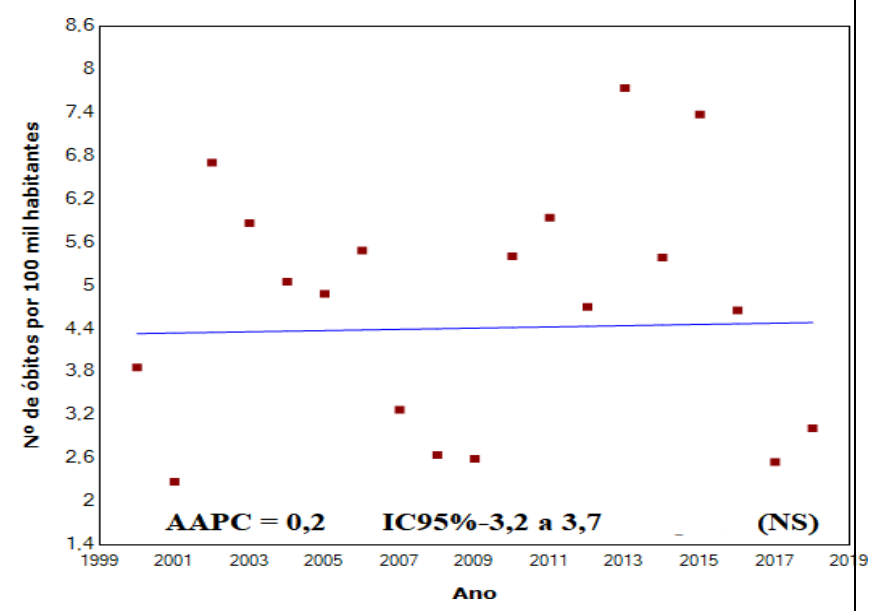

(F) TMFE 50 a 59 anos

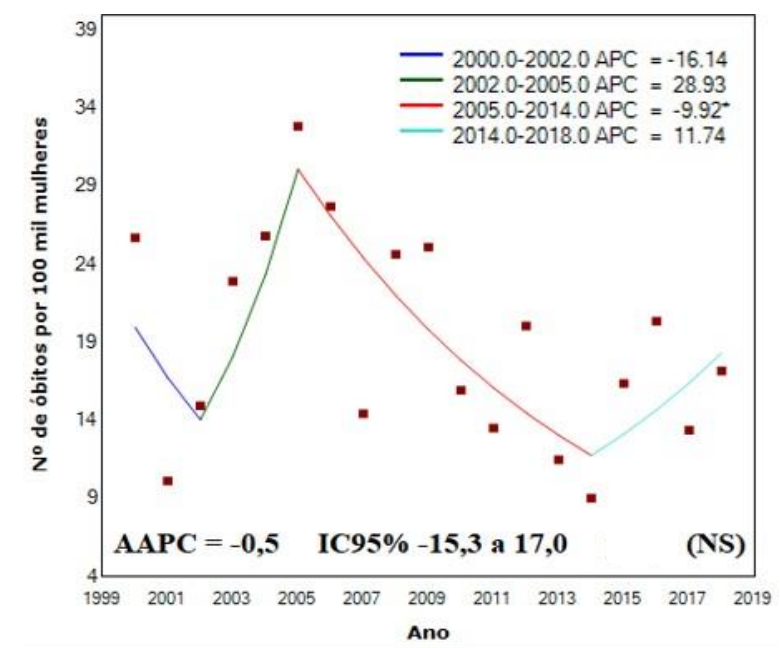



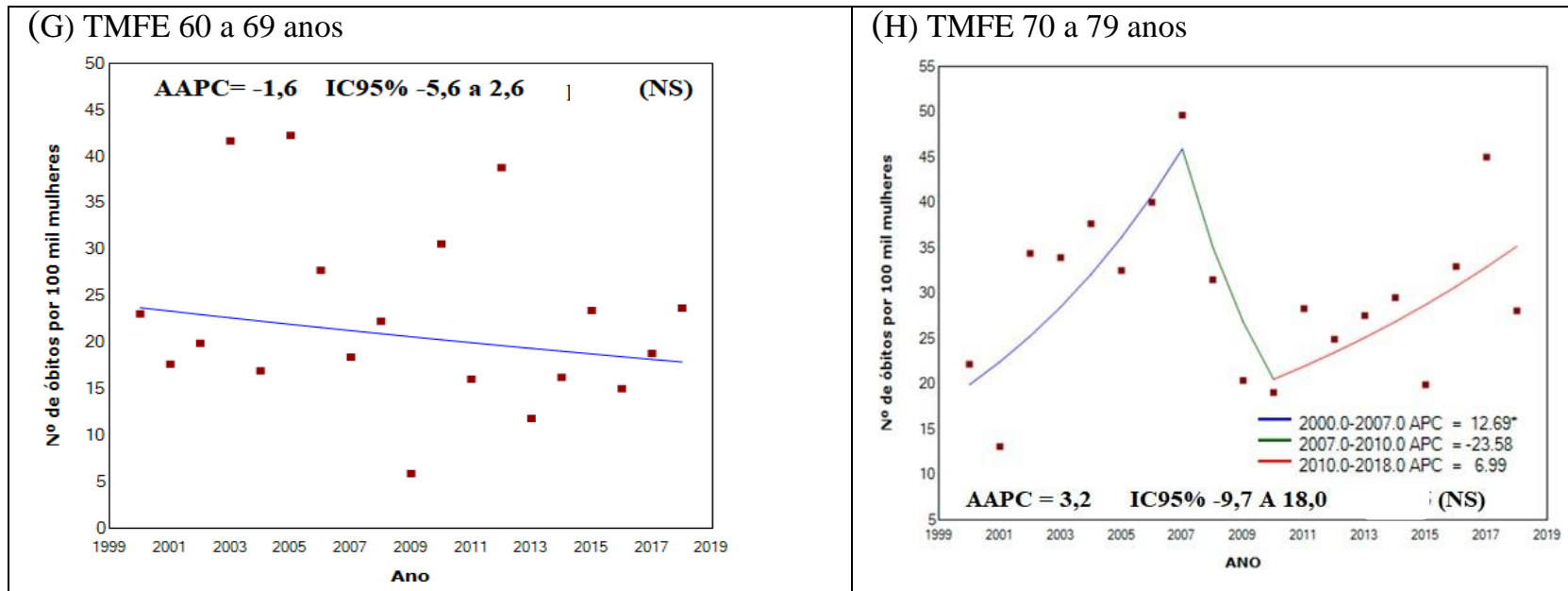

(I) TMFE 80 anos ou mais

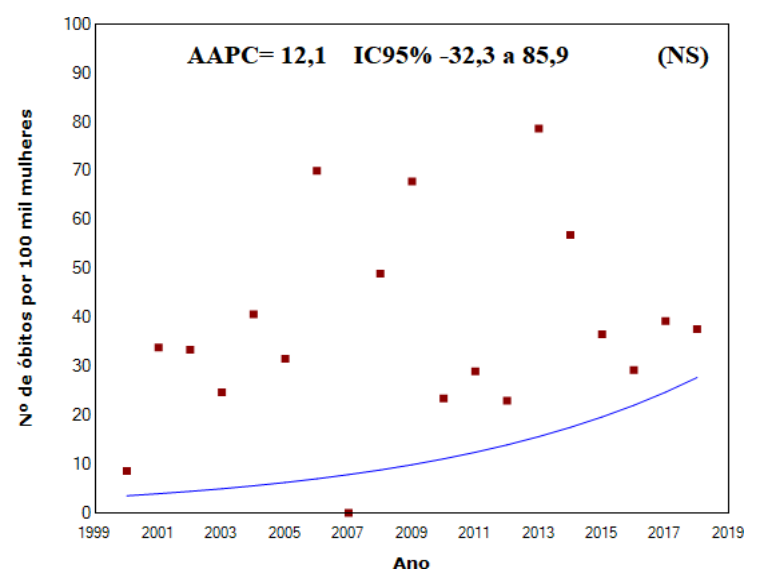

Legenda: AAPC: percentual de variação anual médio (Average Annual Percent Change); APC: percentual de variação anual (Annual Percent Change); NS: não significante estatisticamente. Fonte: DATASUS (2020).

O modelo de regressão mostrou tendência de crescimento da mortalidade tanto na taxa bruta, com um AAPC de 2,1\%e um intervalo de confiança (IC) de 95\% de $-3,8$ a 8,4 (Quadro 9 - A), quanto da padronizada, com um AAPC de $1.0 \%$ e um IC de 95\% entre -0,2 a 2,3 (Quadro 9-B), embora ambas não foram estatisticamente significantes (NS).

Porém, ao analisar a taxa bruta de mortalidade em faixas temporais específicas, verificou-se que de 2000 a 2004 , houve um crescimento estatisticamente significativo de 12,32\% (APC), seguido de um período queda (2004-2007) (NS) e de leve crescimento (2007 a 2018) (NS) (Quadro 9 - A).

Já em relação a faixa etária, destaca-se o resultado encontrado para a faixa de 40 a 49 anos, que com um AAPC de 2,8\% e um IC95\% de $-4,6$ a -1,0, representa diminuição estatisticamente significante na taxa de mortalidade por neoplasia maligna de útero no período de 2000 a 2018 (Quadro 9 - E). Verificou-se ainda queda anual de 9,92\% nas taxas de mortalidade de mulheres de 50 a 59 anos no período de 2005 a 2014 (Quadro 9 - F), bem como crescimento de 12,69\% ao ano desse índice em mulheres de 70 a 79 anos no entre 2000 e 2007 (Quadro 9 - H).

As análises das faixas etárias de 20 a 29 anos (Quadro 9-C), 30 a 39 anos (Quadro 9- D) e 80 anos ou mais (Quadro 9 - I), resultaram em AAPC positivo, indicando tendência de crescimento, contudo como o IC95\% apresentava valores positivos e negativos, os valores de AAPC não são considerados estatisticamente significantes e as tendências temporais de mortalidade são classificadas como estacionárias. Fato semelhante ocorreu na análise para a faixa etária de 60 a 69 anos (Quadro 9-G), indicando uma tendência de diminuição na taxa de mortalidade, embora não seja estatisticamente significante. 


\section{Discussão}

O conhecimento do perfil de mortalidade por câncer de colo de útero contribui para a identificação dos grupos de maior risco dessa neoplasia. Neste estudo, foram observados os maiores números de óbitos em mulheres na faixa etária de 50-59 anos, de cor parda, solteiras, com nenhuma escolaridade e que ocorreram em ambiente hospitalar. Além disso, ao analisar o percentual da realização dos exames citopatológicos em mulheres residentes em Sergipe entre os anos de 2006 e 2018, encontrou-se tendência de aumento (NS) nas faixas etárias de 15 a 19 anos e 60 anos ou mais, e de queda (NS) nas demais faixas etárias.

Tallon et al. (2020), ao avaliarem a mortalidade entre os anos de 2012-2016 nas mulheres entre 25 e 64 anos, também encontraram tendência crescente com 18.574 óbitos (67,02\%), apresentado maior percentual de mortes na faixa etária de 50-54 anos (11,52\%). Fato que, assim como o encontrado no presente estudo, pode ser justificado, dentre outros fatores, pelo longo tempo entre a contaminação pelo HPV e o desenvolvimento do câncer de colo de útero, resultando em aumento da ocorrência da neoplasia com o avanço da idade.

Neste estudo, o maior percentual de óbitos foi encontrado na cor/raça parda (51,49\%). Esse resultado é justificado devido a influência das diferentes etnias encontradas no país. Um dado importante a ser observado é que a cor preta/parda possui a pior sobrevida para o câncer de colo de útero quando comparada à cor branca, provavelmente devido a desigualdades existentes entre os grupos e contexto de vulnerabilidades sociais (Tsuchiya et al., 2017).

Outro determinante de mortalidade refere-se ao número de óbitos em mulheres com escolaridade menor que sete anos. No Estado de Santa Catarina, resultados evidenciam que as mulheres com escolaridade de doze anos ou mais possuíram menor índice de mortalidade (6\%) quando comparadas às mulheres com escolaridade entre quatro a sete anos (31\%), uma vez que a baixa escolaridade pode interferir de forma negativa no acesso das usuárias aos serviços de prevenção existentes (Ribeiro \& Andrade, 2016).

No entanto, é importante destacar que em Sergipe, 25,74\% dos dados registrados possuíram o campo escolaridade "ignorado", o que representa falha no sistema de vigilância em saúde, impedindo, de forma significativa, a identificação da situação epidemiológica do Estado nesse período para essa variável.

Outros fatores de risco que contribuem para o desenvolvimento do câncer de colo útero são a multiplicidade de parceiros, o uso prolongado de pílulas anticoncepcionais, tabagismo, história de doenças sexualmente transmissíveis e sexarca precoce (Inca, 2020b).

Ao avaliar a evolução das taxas de mortalidade do período, verificou-se uma tendência de crescimento na maioria das taxas de mortalidade, contudo esses dados não foram significantes estatisticamente, e foram considerados estacionárias. Esses resultados podem ser justificados pelos investimentos em programas e políticas públicas para rastreamento, prevenção e tratamento precoce dessa neoplasia, que mesmo com o crescimento populacional no período em estudo, reduz a incidência e mortalidade por câncer do colo do útero (Madeiro, 2016).

Ainda, nos resultados encontrados, considerando a variação percentual média da taxa de cobertura de exames citopatológicos realizados entre 2006 e 2018, verifica-se tendência de crescimento (NS) nas faixas etárias de 15 a 19 anos e 60 anos ou mais, e tendência de queda (NS) nas faixas etárias centrais, de 20 a 49 anos, cujo valor foi praticamente nulo na faixa de 50 a 59 anos (NS).

O modelo de análise ainda permitiu observar que nos anos iniciais do estudo houve maiores taxas de cobertura, isso se deve, provavelmente, ao reflexo da implementação da Política Nacional de Atenção Oncológica (PNAO). Essa política trouxe como componente essencial, previsto nos planos estaduais e municipais de saúde, o controle dos cânceres do colo do útero e de mama e do Pacto pela Saúde (2006), através da detecção precoce. A queda apresentada posteriormente pode representar tanto a diminuição da disseminação de informações para incentivo e na propaganda, minimizando a discussão 
sobre política, quanto uma falha no gerenciamento do sistema de informação (Herget et al., 2020). O aumento dos exames realizados na faixa etária entre 15 a 19 anos não é uma situação esperada, pois, sabe-se que a doença apresenta um desenvolvimento lento, e as evidências demonstram que alterações cervicais nesta idade geralmente evoluem com remissão espontânea. Por estes motivos, entrou-se em consenso sobre a periodicidade e faixa etária preconizada para o exame citopatológico em que não se recomenda a realização em menores de 20 anos. Isso se concretiza ao verificar a baixa incidência de mortalidade nessa faixa etária no estado de Sergipe (Herget et al., 2020).

Além disso, observou-se que, apesar de ter existido aumento nos exames realizados em mulheres de 60 anos ou mais, este ainda sim é pouco significativo. Este achado corrobora com resultados de outros estudos, que mostram que mulheres com idades nessa faixa etária possuem menos conhecimentos sobre a realização e finalidade do exame citopatológico e o realizam com uma frequência menor. Esse dado ainda é reforçado ao observar que algumas mulheres de idade mais avançada discernem o exame apenas como curativo, associando-o às Infecções Sexualmente Transmissíveis, e não como um procedimento de prevenção (Barreto et al., 2018).

A maioria das mulheres com 60 anos ou mais, por não terem um esclarecimento significativo em relação a periodicidade do rastreamento do câncer do colo uterino, acabam se tornando mais propensas a desenvolverem a patologia, uma vez que, o desconhecimento de informações pode estar relacionado com a ausência de vínculo e comunicação entre a usuária e profissional de saúde, provocando consequentemente um grande desafio para os serviços de saúde pública (Herget et al., 2020).

Já na faixa etária central de 20 a 49 anos, observa-se uma tendência de queda (NS). As mulheres com idade de 20 a 49 anos compõem faixa de risco para o câncer de colo de útero. Essa queda pode ser atribuída ao fato de muitas delas apresentarem vergonha e medo da realização do exame citopatológico, além de outros aspectos anteriormente citados, que provém de experiências negativas, tanto de terceiros como suas próprias em coletas anteriores, além do medo da possibilidade de receberem resultado positivo para o câncer (Leite et al., 2018).

O medo dispõe-se como um fator negativo na realização, pois, esse sentimento, durante a coleta, faz com que algumas mulheres retardem a realização do exame preventivo. Esse fato pode ser atribuído a uma possível falha na conscientização da população da rede de saúde pública, o que muitas vezes prejudica a aceitação ao exame como importante método preventivo (Tallon et al., 2020).

A educação em saúde constitui uma tarefa essencial no nível da atenção básica. A equipe que compõe a Estratégia de Saúde da Família deve proporcionar educação continuada a fim de eliminar as barreiras existentes sobre a não realização do exame, conscientizando às mulheres sobre a importância da sua realização de forma precoce, para colaborar num possível tratamento (Iglesias et al., 2019). Por isso, é essencial que os profissionais de saúde possibilitem meios para tentar minimizar esse sentimento de medo e vergonha durante a realização do exame, demonstrando empatia e fazendo com que a usuária se sinta o mais à vontade possível, evitando desistências e uma possível complicação do seu estado clínico (Silva et al., 2018).

Sendo assim, a Enfermagem pode interceder de forma positiva nesse cenário, criando rodas de conversas com as mulheres, tanto nas unidades básicas como em locais de atividades socioculturais dos bairros, fazendo uso da educação permanente para conscientiza-las sobre a importância da realização do exame, assim como, sobre o procedimento em si, enfatizando o profissionalismo do responsável por realizá-lo independente do gênero, minimizando dessa forma a hostilidade existente (Oliveira et al., 2019).

O nível de entendimento entre as mulheres sobre a doença e o exame de citologia oncótica ainda é muito pequena, isso se transcreve pela busca dos serviços apenas quando há alguma queixa ou sintomas e não pela prevenção. A ignorância em relação ao exame é mais comum em mulheres com nível de escolaridade mais baixo, pois o acesso a informação é deficiente e com isso a procura dos exames se torna reduzida. Respectivamente detecta-se que mulheres com baixa 
escolaridade são mais suscetíveis as infecções, por desconhecerem a doença e o exame, além da maior dificuldade da manutenção da saúde, voltada às medidas preventivas e de controle (Onofre et al., 2019).

Destaca-se ainda o estado civil onde presume-se que mulheres casadas possuem parceiro fixo, porém, possuem vida sexual mais ativa e fazem menos uso de medidas preventivas quando comparadas as solteiras e viúvas. Portanto, ressalta-se a importância da periodicidade na realização do exame nessa população (Leite et al., 2018).

Com isso, observa-se a necessidade de intervenções que ampliem a cobertura do exame citopatológico, pois, se tratando do câncer do colo de útero, a detecção precoce é a estratégia fundamental para o seu controle (Leite et al., 2018).

Além da estratégia organizada, a coleta do exame de forma oportunística também pode ser eficiente no aumento da cobertura, já que busca englobar populações que estão descobertas, visando lhes garantir o rastreio e tratamento oportuno. Seja durante outro atendimento de saúde e/ou por meio de ação específica, como a coleta em unidade móvel, o rastreamento oportunístico pode ser um aliado para constituir um primeiro contato e vínculo com mais mulheres, colaborando também para a coleta de dados e informações que permitirão uma investigação global da cobertura de exames, colaborando na gestão da estratégia organizada (Oliveira et al., 2018).

Outra estratégia importante é a disseminação do rastreio por múltiplos canais de comunicação e de materiais com linguagem de fácil entendimento para ao público alvo intensificando o impacto das estratégias de prevenção do câncer informando a população, esclarecendo mitos relacionados ao exame e atraindo principalmente as mulheres que não estão em dia com o rastreio (Ribeiro et al., 2016).

\section{Conclusão}

Este estudo permitiu identificar a possível relação entre a mortalidade por câncer de colo de útero no Estado de Sergipe a realização de exames citopatológicos. De maneira geral, houve tendência de crescimento da mortalidade no período estudado e tendência de diminuição da taxa de cobertura. Contudo, percebe-se que, mesmo tais resultados não tendo sido estatisticamente significantes, tais índices podem representar variáveis inversamente proporcionais.

Observou-se também que apesar de existir avanços na atenção primária, o câncer do colo do útero ainda é um desafio a ser vencido, uma vez que pode estar associado à educação e a outros determinantes sociais. Ademais, percebe-se o baixo número de exames citopatológicos realizados quando comparados a quantidade de mulheres em idade fértil residentes em Sergipe, o que pode ser fruto da baixa disponibilidade da realização dos exames ou baixa procura/adesão por parte das mulheres.

A limitação desta pesquisa se deu pela baixa quantidade de estudos atuais encontrados acerca da temática que subsidiem a influência das variáveis sociodemográficas, o que demonstra que este tema ainda é de pouca visibilidade e baixo interesse no meio acadêmico, embora seja de extrema relevância e apresentar-se como importante problema de saúde pública.

Outra limitação encontrada foi a insipiência de dados no sistema de informação, que comprometeu significativamente a análise epidemiológica de algumas variáveis sociodemográficas. Isso pode ser reflexo do registro ineficaz das informações pelos profissionais assistenciais e, portanto, devem ser observados pela equipe gestora.

Além disso, a propagação do conhecimento sobre a realização do exame citopatológico, o uso do preservativo e da imunização como forma de prevenção da infecção por HPV, bem como, a busca por mulheres que não comparecem as unidades de saúde para realização do exame, são ferramentas que podem auxiliar a diminuição das taxas de mortalidade por neoplasias uterinas.

Diante disso, mostra-se a importância da realização de trabalhos futuros para melhores investigações acerca do assunto, bem como a construção de ações voltadas para a atenção primária e a busca ativa a fim de desenvolver os resultados 
dos coeficientes de exames preventivos, contribuindo assim no campo social e repassando informações pertinentes que possam minimizar a incidência e a prevalência da doença, assim como, divulgar a importância da adesão ao exame e então reduzir as taxas de óbito.

\section{Referências}

Barreto, A. M. M. A., Oliveira, F. M. C., \& Gomes, M. Q. C. (2018). Intervenção educativa em saúde para idosas à cerca do exame Papanicolau. Revista Online de Pesquisa: Cuidado é Fundamental, 10, 252-254.

Carvalho, J. R. A. (2019). Prevalência do câncer de colo do útero e lesões intraepiteliais: População assistida em mutirões de prevenção ao câncer. Revista Unimontes Científicas.

Carvalho, P. G., O’Dwer, G., \& Rodrigues, N. C. P. (2018). Trajetórias assistenciais de mulheres entre diagnóstico e início de tratamento do câncer de colo uterino. Saúde Em Debate, 42(118), 687-701. https://doi.org/10.1590/0103-1104201811812

Correia, R. A., Bonfim, C. V. do, Ferreira, D. K. da S., Furtado, B. M. A. S. M., Costa, H. V. V. da, Feitosa, K. M. A., \& Santos, S. L. dos. (2018). Qualidade de vida após o tratamento do câncer do colo do útero. Escola Anna Nery, 22(4). https://doi.org/10.1590/2177-9465-ean-2018-0130

Farias, A. C. B., \& Barbieri, A. R. (2016). Seguimento do câncer de colo de útero: Estudo da continuidade da assistência à paciente em uma região de saúde. Escola Anna Nery Revista de Enfermagem, 20(4). https://www.redalyc.org/articulo.oa?id=127746815015

Febrasgo. (2017). Rastreio, Diagnóstico e Tratamento do Câncer de Colo de Útero. Série Orientações e Recomendações FEBRASGO, 1(2), 1-62. https://www.febrasgo.org.br/media/k2/attachments/05Z-ZDIAGNOySTICOZRASTREIOZEZTRATAMENTOZDOZCAyNCERZDEZCOL

OZDEZUyTERO.pdf

Herget, A. R., Bueno, A. C. R., \& Santos, A. de L. (2020). Análise dos coeficientes de exames citopatológicos realizados e alterados no Paraná. Revista de Pesquisa Cuidado é Fundamental Online, 12, 1125-1131. https://doi.org/10.9789/2175-5361.rpcfo.v12.8011

Iglesias, G. A., Larrubia, L. G., Campos Neto, A. de S., Pacca, F. C., \& Iembo, T. (2019). Conhecimento e adesão ao Papanicolau de mulheres de uma rede de Atenção Primária à Saúde. Revista de Ciências Médicas, 28(1), 21. https://doi.org/10.24220/2318-0897v28n1a4008

INCA. (2016). Coordenação de Prevenção e Vigilância. Divisão de Detecção Precoce e Apoio à Organização de Rede. Diretrizes brasileiras para o rastreamento do câncer do colo do útero.

Leite, K. N. S., da Silva, J. P., de Sousa, K. M., Rodrigues, S. da C., de Souza, T. A., Alves, J. P., de Souza, A. R. D., \& Rodrigues, A. R. da S. (2018). Exame Papanicolau: fatores que influenciam a não realização do exame em mulheres de 40 a 65 anos. Arquivos de Ciências Da Saúde, 25(2), 15-19. http://www.cienciasdasaude.famerp.br/index.php/racs/article/view/9334

Madeiro, A. et al. (2016). Tendências da mortalidade por câncer do colo do útero no Piauí, 2000-2011. Caderno Saúde Coletiva, 24(3), 282-285.

Oliveira, D. D. S., Sá, A. V., Gramacho, R. D. C. C. V., Silva, R. D. C. V. da, \& Oliveira, J. D. S. (2019). Atuação da enfermeira frente aos fatores que interferem na adesão de mulheres idosas ao exame de Papanicolau. Revista Enfermagem Contemporânea, 8(1), 87. https://doi.org/10.17267/23173378rec.v8i1.2155

Oliveira, M. M., Andrade, S. S. C. A., Oliveira, P. P. V., Silva, G. A. E., Silva, M. M. A., \& Malta, D. C. (2018). Cobertura de exame Papanicolau em mulheres de 25 a 64 anos, segundo a Pesquisa Nacional de Saúde e o Sistema de Vigilância de Fatores de Risco e Proteção para Doenças Crônicas por Inquérito Telefônico, 2013. Revista Brasileira de Epidemiologia, 21, 1-11. https://doi.org/10.1590/1980-549720180014

Onofre, M. F., Vieira, R. D., \& Bueno, G. H. (2019). Principais fatores que dificultam a adesão ao exame de citologia oncótica: Uma Revisão de Literatura. Enfermagem Revista, 22(2), 231-239.

Pereira, A. S., Shitsuka, D. M., Parreira, F. J., \& Shitsuka, R. (2018). Metodologia da pesquisa científica. In Metodologia da Pesquisa Científica. https://repositorio.ufsm.br/bitstream/handle/1/15824/Lic_Computacao_Metodologia-Pesquisa-Cientifica.pdf?sequence=1. Acesso em: 28 março 2020.

Ribeiro, J. C., \& Andrade, S. R. de. (2016). Vigilância em saúde e a cobertura de exame citopatológico do colo do útero: revisão integrativa. Texto Contexto Enfermagem, 25(4), 1-12. http://www.revenf.bvs.br/scielo.php?script=sci_arttext\&pid=S0104-07072016000400501

Ribeiro, K. P., Zocche, D. A. A., Hahn, E., Silva, J. R., \& Barbisan, L. (2016). O perfil da mortalidade por câncer do colo do útero, no período de 2010 a 2015, no Estado de Santa Catarina. Brazilian Journal of Surgery and Clinical Research - BJSCR, 15(4), 11-12. http://www.mastereditora.com.br/bjscr

Silva, M. A., De Freitas, H. G., Ribeiro, R. L., Oliveira, M. N. L., Sanches, F. C. de A., \& Thuler, L. C. S. (2018). Fatores que, na Visão da Mulher, Interferem no Diagnóstico Precoce do Câncer do Colo do Útero. Revista Brasileira de Cancerologia, 64(1), 99-106. https://doi.org/10.32635/21769745.rbc.2018v64n1.124

Tallon, B., Monteiro, D., Soares, L., Rodrigues, N., \& Morgado, F. (2020). Tendências da mortalidade por câncer de colo no Brasil em 5 anos (2012-2016). Saúde Em Debate, 44(125), 362-371. https://doi.org/10.1590/0103-1104202012506

Tsuchiya, C., Lawrence, T., Klen, M., Fernandes, R., \& Alves, M. R. (2017). O câncer de colo do útero no Brasil: uma retrospectiva sobre as políticas públicas voltadas à saúde da mulher. Jornal Brasileiro de Economia Da Saúde, 9(1), 137-147. https://doi.org/10.21115/jbes.v9.n1.p137-47 